\title{
Two approaches for describing the Casimir interaction in graphene: Density-density correlation function versus polarization tensor
}

\author{
G. L. Klimchitskaya, V.M. Mostepanenko and Bo Sernelius
}

\section{Linköping University Post Print}

\section{Tweet}

N.B.: When citing this work, cite the original article.

Original Publication:

G. L. Klimchitskaya, V.M. Mostepanenko and Bo Sernelius, Two approaches for describing the Casimir interaction in graphene: Density-density correlation function versus polarization tensor, 2014, Physical Review B. Condensed Matter and Materials Physics, (89), 12, 125407. http://dx.doi.org/10.1103/PhysRevB.89.125407

Copyright: American Physical Society http://www.aps.org/

Postprint available at: Linköping University Electronic Press http://urn.kb.se/resolve?urn=urn:nbn:se:liu:diva-105570 


\title{
Two approaches for describing the Casimir interaction in graphene: Density-density correlation function versus polarization tensor
}

\author{
G. L. Klimchitskaya, ${ }^{1,2}$ V. M. Mostepanenko, ${ }^{1,2}$ and Bo E. Sernelius ${ }^{3}$ \\ ${ }^{1}$ Central Astronomical Observatory at Pulkovo of the Russian Academy of Sciences, St. Petersburg 196140, Russia \\ ${ }^{2}$ Institute of Physics, Nanotechnology and Telecommunications, St. Petersburg State Polytechnical University, St. Petersburg 195251, Russia \\ ${ }^{3}$ Division of Theory and Modeling, Department of Physics, Chemistry and Biology, Linköping University, Linköping SE-581 83, Sweden
}

(Received 24 January 2014; revised manuscript received 20 February 2014; published 6 March 2014)

\begin{abstract}
A comparison study of theoretical approaches to the description of the Casimir interaction in layered systems including graphene is performed. It is shown that at zero temperature, the approach using the polarization tensor leads to the same results as the approach using the longitudinal density-density correlation function of graphene. An explicit expression for the zero-temperature transverse density-density correlation function of graphene is provided. We further show that the computational results for the Casimir free energy of graphene-graphene and graphene-Au plate interactions at room temperature, obtained using the temperature-dependent polarization tensor, deviate significantly from those using the longitudinal density-density correlation function defined at zero temperature. We derive both the longitudinal and transverse density-density correlation functions of graphene at nonzero temperature. The Casimir free energy in layered structures including graphene, computed using the temperature-dependent correlation functions, is exactly equal to that found using the polarization tensor.
\end{abstract}

DOI: 10.1103/PhysRevB.89.125407

PACS number(s): 78.67.Wj, 65.80.Ck, 12.20.-m, 42.50.Ct

\section{INTRODUCTION}

During the last few years, due to their remarkable properties, graphene and other carbon-based nanostructures have attracted the particular attention of many experimentalists and theorists [1,2]. These investigations have provided further impetus to technological progress. One of the topical subjects, which came to the experimental attention very recently [3], is the van der Waals and Casimir interaction of graphene deposited on a substrate with the test body made of an ordinary material.

Theorists have already undertook a number of studies of graphene-graphene and graphene-material plate interactions using the Dirac model of graphene [1], which assumes the linear dispersion relation for the graphene bands at low energies. Specifically, in Ref. [4], the van der Waals coefficient for two graphene sheets at zero temperature was calculated using the correlation energy from the random-phase approximation (in Ref. [5], the obtained value was improved using the nonlocal dielectric function of graphene). In Ref. [6], the van der Waals and Casimir forces between graphene and the ideal-metal plane were calculated at zero temperature using the Lifshitz theory, where the reflection coefficients of the electromagnetic oscillations were expressed via the polarization tensor in $(2+1)$ dimensions. Important progress was achieved in Ref. [7], where the force at nonzero temperature between two graphene sheets and between a graphene and a material plate was expressed via the Coulomb coupling between density fluctuations. The density-density correlation function in the random-phase approximation has been used. It was shown [7] that for graphene the relativistic effects are not essential, and that the thermal effects become crucial at much shorter separations than in the case of ordinary materials. In Ref. [8], the graphene-graphene interaction was computed under an assumption that the conductivity of graphene can be described by the in-plane optical properties of graphite. It was shown [9] that for a sufficiently large band-gap parameter of graphene the thermal Casimir force can vary severalfold with temperature. In Ref. [10], the reflection coefficients in the Lifshitz theory were expressed via the polarization tensor at nonzero temperature, whose components were explicitly calculated. The detailed computations of graphene-graphene and graphene-real-metal Casimir interactions using this method were performed [10-12]. Finally, in Ref. [13], the reflection coefficients of the Lifshitz theory were generalized for the case of planar structures including two-dimensional sheets. The graphene-graphene and graphene-real-metal interactions at both zero and nonzero temperature were computed by using the electric susceptibility (polarizability) of graphene expressed via the density-density correlation function. It was argued [13] that the zero-temperature form of polarizability can be used also at room temperature.

We underline that there is no complete agreement between the results of different papers devoted to the van der Waals and Casimir interactions with graphene (see Ref. [12] where some of the results obtained are compared). In fact, all of the approaches go back to the Lifshitz theory [14-16], but with different approximations made and with various forms of the reflection coefficients used. By and large, the approaches based on the density-density correlation function used its longitudinal version, i.e., neglected by the role of (small [7]) relativistic effects. Furthermore, dependence of the correlation function on temperature, which was unknown until the present time, was obtained by means of scaling [7] or even neglected [13]. By contrast, calculations based on the polarization tensor are fully relativistic and include an explicit dependence of its components on the temperature [10-12]. This is the reason why it would be useful to establish a link between the two approaches and to test the validity of the approximations used.

In this paper, we find a correspondence between the reflection coefficients of the electromagnetic fluctuations on graphene expressed in terms of electric susceptibility (polarizability) of graphene and components of the polarization tensor. On this basis, we derive explicit expressions for both longitudinal and transverse electric susceptibilities of graphene, density-density correlation function, and 
conductivities at arbitrary temperature. Then we consider the limiting cases of the obtained expressions at zero temperature and find that the longitudinal version coincides with that derived within the random phase approximation. Furthermore, we compare the computational results for graphene-graphene and graphene-real-metal interactions at room temperature obtained using the polarization tensor [11,12] with those obtained using the density-density correlation function in Ref. [13]. In doing so, we pay special attention to contributions of the transverse electric susceptibility of graphene and explicit temperature dependence of the longitudinal density-density correlation function to the Casimir free energy.

The paper is organized as follows. In Sec. II, we establish a link between the two approaches and derive the density-density correlation functions at nonzero temperature. Section III is devoted to the case of zero temperature. In Sec. IV, the computational results for graphene-graphene and graphene-real-metal thermal Casimir interactions using the zero-temperature correlation function and the polarization tensor at room temperature are compared. In Sec. V, the reader will find our conclusions and discussion.

\section{COMPARISON BETWEEN THE REFLECTION COEFFICIENTS IN TWO THEORETICAL APPROACHES}

As discussed in Sec. I, all theoretical approaches to the van der Waals and Casimir interaction between two graphene sheets or between graphene and a material plate go back to the Lifshitz theory representing the free energy per unit area at temperature $T$ in thermal equilibrium in the form [14-16]

$$
\begin{aligned}
\mathcal{F}(a, T)= & \frac{k_{B} T}{2 \pi} \sum_{l=0}^{\infty} \int_{0}^{\infty} k_{\perp} d k_{\perp} \\
& \times\left\{\ln \left[1-r_{\mathrm{TM}}^{(1)}\left(i \xi_{l}, k_{\perp}\right) r_{\mathrm{TM}}^{(2)}\left(i \xi_{l}, k_{\perp}\right) e^{-2 a q_{l}}\right]\right. \\
& \left.+\ln \left[1-r_{\mathrm{TE}}^{(1)}\left(i \xi_{l}, k_{\perp}\right) r_{\mathrm{TE}}^{(2)}\left(i \xi_{l}, k_{\perp}\right) e^{-2 a q_{l}}\right]\right\} .
\end{aligned}
$$

Here, $k_{B}$ is the Boltzmann constant, $k_{\perp}$ is the projection of the wave vector on the plane of graphene, $\xi_{l}=2 \pi k_{B} T l / \hbar$ with $l=0,1,2, \ldots$ are the Matsubara frequencies, $q_{l}=\left(k_{\perp}^{2}+\right.$ $\left.\xi_{l}^{2} / c^{2}\right)^{1 / 2}$, and the prime on the summation sign indicates that the term with $l=0$ is divided by two. The reflection coefficients on the two boundary planes separated by the vacuum gap of width $a$ for the two independent polarizations of the electromagnetic field, transverse magnetic (TM) and transverse electric (TE), are notated as $r_{\mathrm{TM}, \mathrm{TE}}^{(1)}$ and $r_{\mathrm{TM}, \mathrm{TE}}^{(2)}$.

Let the first boundary plane be the freestanding graphene. There are two main representations for the reflection coefficients $r_{\mathrm{TM}, \mathrm{TE}}^{(1)} \equiv r_{\mathrm{TM}, \mathrm{TE}}^{(g)}$ on graphene. We begin with the TM coefficient. Within the first theoretical approach, the longitudinal electric susceptibility (polarizability) of graphene at the imaginary Matsubara frequencies is expressed as

$$
\alpha^{\|}\left(i \xi_{l}, k_{\perp}\right) \equiv \varepsilon^{\|}\left(i \xi_{l}, k_{\perp}\right)-1=-\frac{2 \pi e^{2}}{k_{\perp}} \chi^{\|}\left(i \xi_{l}, k_{\perp}\right),
$$

where $\chi^{\|}\left(i \xi_{l}, k_{\perp}\right)$ is the longitudinal density-density correlation function. The latter is connected with the dynamical conductivity of graphene by [13]

$$
\sigma^{\|}\left(i \xi_{l}, k_{\perp}\right)=-\frac{e^{2} \xi_{l}}{k_{\perp}^{2}} \chi^{\|}\left(i \xi_{l}, k_{\perp}\right),
$$

where $e$ is the electron charge. Then the TM reflection coefficient of the electromagnetic oscillations on graphene can be expressed as $[13,17,18]$

$$
r_{\mathrm{TM}}^{(g)}\left(i \xi_{l}, k_{\perp}\right)=\frac{q_{l} \alpha^{\|}\left(i \xi_{l}, k_{\perp}\right)}{k_{\perp}+q_{l} \alpha^{\|}\left(i \xi_{l}, k_{\perp}\right)} .
$$

The explicit form for $\alpha^{\|}$is discussed below.

Within the second theoretical approach, the TM reflection coefficient is expressed via the 00 -component $\Pi_{00}$ of the polarization tensor in (2+1)-dimensional space-time [10-12],

$$
r_{\mathrm{TM}}^{(g)}\left(i \xi_{l}, k_{\perp}\right)=\frac{q_{l} \Pi_{00}\left(i \xi_{l}, k_{\perp}\right)}{2 \hbar k_{\perp}^{2}+q_{l} \Pi_{00}\left(i \xi_{l}, k_{\perp}\right)} .
$$

The analytic expression for $\Pi_{00}$ is known [10-12]. It depends on the temperature both implicitly (through the Matsubara frequencies) and explicitly, as on a parameter. For the pristine (undoped) gapless graphene, one has [10-12]

$$
\begin{aligned}
\Pi_{00}\left(i \xi_{l}, k_{\perp}\right) & \\
= & \frac{\pi \hbar \alpha k_{\perp}^{2}}{f\left(\xi_{l}, k_{\perp}\right)}+\frac{8 \hbar \alpha c^{2}}{v_{F}^{2}} \int_{0}^{1} d x\left\{\frac{k_{B} T}{\hbar c}\right. \\
& \times \ln \left[1+2 \cos (2 \pi l x) e^{-\theta_{T}\left(\xi_{l}, k_{\perp}, x\right)}+e^{-2 \theta_{T}\left(\xi_{l}, k_{\perp}, x\right)}\right] \\
& -\frac{\xi_{l}}{2 c}(1-2 x) \frac{\sin (2 \pi l x)}{\cosh \theta_{T}\left(\xi_{l}, k_{\perp}, x\right)+\cos (2 \pi l x)} \\
& \left.+\frac{\xi_{l}^{2} \sqrt{x(1-x)}}{c^{2} f\left(\xi_{l}, k_{\perp}\right)} \frac{\cos (2 \pi l x)+e^{-\theta_{T}\left(\xi_{l}, k_{\perp}, x\right)}}{\cosh \theta_{T}\left(\xi_{l}, k_{\perp}, x\right)+\cos (2 \pi l x)}\right\},
\end{aligned}
$$

where $\alpha=e^{2} /(\hbar c)$ is the fine-structure constant, $v_{F}$ is the Fermi velocity, and the following notations are introduced:

$$
\begin{aligned}
f\left(\xi_{l}, k_{\perp}\right) & \equiv\left(\frac{v_{F}^{2}}{c^{2}} k_{\perp}^{2}+\frac{\xi_{l}^{2}}{c^{2}}\right)^{1 / 2}, \\
\theta_{T}\left(\xi_{l}, k_{\perp}, x\right) & \equiv \frac{\hbar c}{k_{B} T} f\left(\xi_{l}, k_{\perp}\right) \sqrt{x(1-x)} .
\end{aligned}
$$

Now we equate the right-hand sides of Eqs. (4) and (5) and obtain the expression for the longitudinal polarizability of graphene at nonzero temperature via the 00 component of the polarization tensor,

$$
\alpha^{\|}\left(i \xi_{l}, k_{\perp}\right)=\frac{1}{2 \hbar k_{\perp}} \Pi_{00}\left(i \xi_{l}, k_{\perp}\right) .
$$

Using Eq. (2), for the longitudinal density-density correlation function, one obtains

$$
\chi^{\|}\left(i \xi_{l}, k_{\perp}\right)=-\frac{1}{4 \pi e^{2} \hbar} \Pi_{00}\left(i \xi_{l}, k_{\perp}\right),
$$

where $\Pi_{00}$ is given by Eq. (6). Similar to the polarization tensor, the density-density correlation function depends on $T$ both implicitly and explicitly. The longitudinal conductivity of graphene at any $T$ is given by Eq. (3).

We continue with the TE reflection coefficient. Note that Eqs. (2) and (3) remain valid for the transverse quantities: 
the polarizability of graphene $\alpha^{\perp}\left(i \xi_{l}, k_{\perp}\right)$, the transverse permittivity $\varepsilon^{\perp}\left(i \xi_{l}, k_{\perp}\right)$, the density-density correlation function $\chi^{\perp}\left(i \xi_{l}, k_{\perp}\right)$, and the conductivity $\sigma^{\perp}\left(i \xi_{l}, k_{\perp}\right)$. The TE reflection coefficient on graphene in terms of the transverse polarizability was found in Ref. [13],

$$
r_{\mathrm{TE}}^{(g)}\left(i \xi_{l}, k_{\perp}\right)=-\frac{\xi_{l}^{2} \alpha^{\perp}\left(i \xi_{l}, k_{\perp}\right)}{c^{2} k_{\perp} q_{l}+\xi_{l}^{2} \alpha^{\perp}\left(i \xi_{l}, k_{\perp}\right)} .
$$

Note that according to our knowledge no explicit expression of $\alpha^{\perp}$ for graphene is available in the published literature.
In terms of the polarization tensor, the TE reflection coefficient takes the form [10-12]

$$
r_{\mathrm{TE}}^{(g)}\left(i \xi_{l}, k_{\perp}\right)=-\frac{k_{\perp}^{2} \Pi_{\mathrm{tr}}\left(i \xi_{l}, k_{\perp}\right)-q_{l}^{2} \Pi_{00}\left(i \xi_{l}, k_{\perp}\right)}{2 \hbar k_{\perp}^{2} q_{l}+k_{\perp}^{2} \Pi_{\mathrm{tr}}\left(i \xi_{l}, k_{\perp}\right)-q_{l}^{2} \Pi_{00}\left(i \xi_{l}, k_{\perp}\right)},
$$

where the index tr denotes the sum of spatial components $\Pi_{1}^{1}$ and $\Pi_{2}^{2}$. For the undoped gapless graphene, the analytic expression for $\Pi_{t r}$ is the following [10-12]:

$$
\begin{aligned}
\Pi_{\mathrm{tr}}\left(i \xi_{l}, k_{\perp}\right)= & \Pi_{00}\left(i \xi_{l}, k_{\perp}\right)+\frac{\pi \hbar \alpha}{f\left(\xi_{l}, k_{\perp}\right)}\left[f^{2}\left(\xi_{l}, k_{\perp}\right)+\frac{\xi_{l}^{2}}{c^{2}}\right]+8 \hbar \alpha \int_{0}^{1} d x\left\{\frac{\xi_{l}}{c}(1-2 x) \frac{\sin (2 \pi l x)}{\cosh \theta_{T}\left(\xi_{l}, k_{\perp}, x\right)+\cos (2 \pi l x)}\right. \\
& \left.-\frac{\sqrt{x(1-x)}}{f\left(\xi_{l}, k_{\perp}\right)}\left[f^{2}\left(\xi_{l}, k_{\perp}\right)+\frac{\xi_{l}^{2}}{c^{2}}\right] \frac{\cos (2 \pi l x)+e^{-\theta_{T}\left(\xi_{l}, k_{\perp}, x\right)}}{\cosh \theta_{T}\left(\xi_{l}, k_{\perp}, x\right)+\cos (2 \pi l x)}\right\} .
\end{aligned}
$$

By equating the right-hand sides of Eqs. (10) and (11), one obtains the expression for the transverse polarizability of graphene at any nonzero temperature,

$$
\alpha^{\perp}\left(i \xi_{l}, k_{\perp}\right)=\frac{c^{2}}{2 \hbar k_{\perp} \xi_{l}^{2}}\left[k_{\perp}^{2} \Pi_{\mathrm{tr}}\left(i \xi_{l}, k_{\perp}\right)-q_{l}^{2} \Pi_{00}\left(i \xi_{l}, k_{\perp}\right)\right] .
$$

The respective result for the transverse density-density correlation function is found from an equation similar to Eq. (2),

$\chi^{\perp}\left(i \xi_{l}, k_{\perp}\right)=-\frac{c^{2}}{4 \pi \hbar e^{2} \xi_{l}^{2}}\left[k_{\perp}^{2} \Pi_{\mathrm{tr}}\left(i \xi_{l}, k_{\perp}\right)-q_{l}^{2} \Pi_{00}\left(i \xi_{l}, k_{\perp}\right)\right]$.

Then the transverse conductivity of graphene is given by Eq. (3) where the index $\|$ is replaced with $\perp$.

We emphasize that Eqs. (4), (5) and (10), (11) are the exact consequences of the Maxwell equations and electrodynamic boundary conditions imposed on the two-dimensional (2D) graphene sheet. For this reason, the obtained connections (8), (9) and (13), (14) between the polarizabilities and densitydensity correlation functions for graphene, on the one hand, and the components of the polarization tensor, on the other hand, are the exact ones. Keeping in mind that Eqs. (6) and (12) for the polarization tensor are calculated in the one-loop approximation [10], the specific expressions for the polarizabilities and density-density correlation functions obtained after the substitution of Eqs. (6) and (12) in Eqs. (8), (9) and (13), (14) should be also considered as found in the same approximation. In the next section, we compare them with those contained in the literature.

To conclude this section, we present an explicit expression for the quantity $k_{\perp}^{2} \Pi_{\text {tr }}-q_{l}^{2} \Pi_{00}$ entering the transverse polarizability, the density-density correlation function, and the conductivity of graphene. Substituting $\Pi_{00}$ from Eq. (6) and $\Pi_{\text {tr }}$ from Eq. (12), one obtains, after identical transformations,

$$
\begin{aligned}
k_{\perp}^{2} \Pi_{\mathrm{tr}}\left(i \xi_{l}, k_{\perp}\right)-q_{l}^{2} \Pi_{00}\left(i \xi_{l}, k_{\perp}\right)= & \pi \hbar \alpha k_{\perp}^{2} f\left(\xi_{l}, k_{\perp}\right)-\frac{8 \hbar \alpha c^{2}}{v_{F}^{2}} \int_{0}^{1} d x\left\{\frac{k_{B} T \xi_{l}^{2}}{\hbar c^{3}} \ln \left[1+2 \cos (2 \pi l x) e^{-\theta_{T}\left(\xi_{l}, k_{\perp}, x\right)}+e^{-2 \theta_{T}\left(\xi_{l}, k_{\perp}, x\right)}\right]\right. \\
& -\left[2 f^{2}\left(\xi_{l}, k_{\perp}\right)-\frac{\xi_{l}^{2}}{c^{2}}\right] \frac{\xi_{l}}{2 c}(1-2 x) \frac{\sin (2 \pi l x)}{\cosh \theta_{T}\left(\xi_{l}, k_{\perp}, x\right)+\cos (2 \pi l x)} \\
& \left.+\sqrt{x(1-x)} f^{3}\left(\xi_{l}, k_{\perp}\right) \frac{\cos (2 \pi l x)+e^{-\theta_{T}\left(\xi_{l}, k_{\perp}, x\right)}}{\cosh \theta_{T}\left(\xi_{l}, k_{\perp}, x\right)+\cos (2 \pi l x)}\right\} .
\end{aligned}
$$

This expression is used in the below calculations.

\section{ENERGY OF THE CASIMIR INTERACTION BETWEEN TWO GRAPHENE SHEETS AT ZERO TEMPERATURE}

In the limiting case $T \rightarrow 0$, the summation over the discrete Matsubara frequencies in Eq. (1) is replaced with integration over the imaginary frequency axis, and for two graphene sheets, one arrives at the Casimir energy per unit area,

$$
\begin{aligned}
E(a, T)= & \frac{\hbar}{4 \pi^{2}} \int_{0}^{\infty} k_{\perp} d k_{\perp} \int_{0}^{\infty} d \xi\left\{\ln \left[1-r_{\mathrm{TM}}^{(g)^{2}}\left(i \xi, k_{\perp}\right) e^{-2 a q}\right]\right. \\
& \left.+\ln \left[1-r_{\mathrm{TE}}^{(g)^{2}}\left(i \xi, k_{\perp}\right) e^{-2 a q}\right]\right\} .
\end{aligned}
$$

Here, the reflection coefficients are given by either Eqs. (4) and (10) or (5) and (11), where the discrete frequencies $\xi_{l}$ are replaced with the continuous $\xi$. 
We begin from the contribution of the TM mode, $E_{\mathrm{TM}}$, to the total energy (16). In terms of the polarization tensor, the reflection coefficient $r_{\mathrm{TM}}^{(g)}$ is given by Eqs. (5) and (6) with the notation (7). As can be seen in Eq. (7), the quantity $\theta_{T}\left(\xi_{l}, k_{\perp}, x\right) \rightarrow \infty$ when $T \rightarrow 0$. Because of this, from Eq. (6) at $T=0 \mathrm{~K}$, one obtains [6]

$$
\Pi_{00}\left(i \xi, k_{\perp}\right)=\frac{\pi \hbar \alpha k_{\perp}^{2}}{f\left(\xi, k_{\perp}\right)} .
$$

Using the notation (7), one obtains from Eq. (8) the longitudinal polarizability of graphene at zero temperature,

$$
\alpha^{\|}\left(i \xi, k_{\perp}\right)=\frac{\pi e^{2}}{2 \hbar} \frac{k_{\perp}}{\sqrt{v_{F}^{2} k_{\perp}^{2}+\xi^{2}}},
$$

and from Eq. (9), the respective density-density correlation function,

$$
\chi^{\|}\left(i \xi, k_{\perp}\right)=-\frac{1}{4 \hbar} \frac{k_{\perp}^{2}}{\sqrt{v_{F}^{2} k_{\perp}^{2}+\xi^{2}}} .
$$

The longitudinal conductivity of graphene at $T=0 \mathrm{~K}$ is obtained from Eqs. (3) and (19).

The density-density correlation function (19) at $T=0 \mathrm{~K}$, derived from the polarization tensor, coincides with the classical result $[19,20]$ which was used in the computations of Ref. [13]. Then, for the TM reflection coefficient on graphene at $T=0 \mathrm{~K}$, we obtain one and the same result either from Eqs. (4) and (18) or from Eqs. (5) and (17),

$$
r_{\mathrm{TM}}^{(g)}\left(i \xi, k_{\perp}\right)=\frac{\pi e^{2} \sqrt{c^{2} k_{\perp}^{2}+\xi^{2}}}{2 \hbar c \sqrt{v_{F}^{2} k_{\perp}^{2}+\xi^{2}}+\pi e^{2} \sqrt{c^{2} k_{\perp}^{2}+\xi^{2}}} .
$$

This reflection coefficient coincides with that used in Ref. [13].

We continue by considering the contribution of the TE mode, $E_{\mathrm{TE}}$, to the Casimir energy (16). In terms of the polarization tensor, the reflection coefficient $r_{\mathrm{TE}}^{(g)}$ is given by Eq. (11). The combination of the components of the polarization tensor, $k_{\perp}^{2} \Pi_{\mathrm{tr}}-q_{l}^{2} \Pi_{00}$, entering Eq. (11), is given by Eq. (15). In the limiting case $T \rightarrow 0$, one obtains, from Eq. (15),

$$
k_{\perp}^{2} \Pi_{\text {tr }}\left(i \xi, k_{\perp}\right)-q^{2} \Pi_{00}\left(i \xi, k_{\perp}\right)=\pi \hbar \alpha k_{\perp}^{2} f\left(\xi, k_{\perp}\right) .
$$

Substituting Eq. (21) in Eq. (13), we find the transverse polarizability of graphene at zero temperature,

$$
\alpha^{\perp}\left(i \xi, k_{\perp}\right)=\frac{\pi e^{2} k_{\perp}}{2 \hbar \xi^{2}} \sqrt{v_{F}^{2} k_{\perp}^{2}+\xi^{2}} .
$$

In a similar way, substituting Eq. (21) in Eq. (14), we find the transverse density-density correlation function at $T=0 \mathrm{~K}$,

$$
\chi^{\perp}\left(i \xi, k_{\perp}\right)=-\frac{k_{\perp}^{2}}{4 \hbar \xi^{2}} \sqrt{v_{F}^{2} k_{\perp}^{2}+\xi^{2}} .
$$

The TE reflection coefficient at $T=0 \mathrm{~K}$ is obtained by either substituting Eq. (21) in Eq. (11) or Eq. (22) in Eq. (10). The

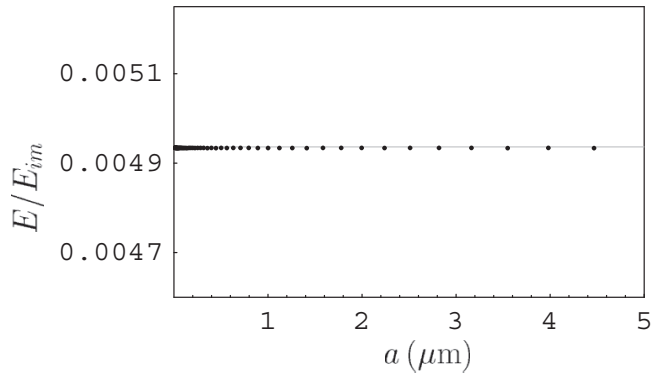

FIG. 1. The Casimir energy per unit area of two graphene sheets at zero temperature normalized to that of two ideal-metal planes is computed using the longitudinal density-density correlation function (dots) and by the polarization tensor (solid line), as functions of separation.

result is

$$
r_{\mathrm{TE}}^{(g)}\left(i \xi, k_{\perp}\right)=-\frac{\pi e^{2} \sqrt{v_{F}^{2} k_{\perp}^{2}+\xi^{2}}}{2 \hbar c \sqrt{c^{2} k_{\perp}^{2}+\xi^{2}}+\pi e^{2} \sqrt{v_{F}^{2} k_{\perp}^{2}+\xi^{2}}} .
$$

As is seen from the comparison of Eqs. (20) and (24), the reflection coefficient $r_{\mathrm{TE}}^{(g)}$ has the opposite sign, as compared with $r_{\mathrm{TM}}^{(g)}$, and its magnitude is obtained from the latter by the interchanging of $c$ and $v_{F}$.

Now we compare the computational results for the Casimir energy per unit area of two parallel graphene sheets at zero temperature obtained in Ref. [13] by means of the density-density correlation function and here using the polarization tensor. In both cases, the Fermi velocity $v_{F}=8.73723 \times 10^{5} \mathrm{~m} / \mathrm{s}$ is employed [13,21,22]. In Fig. 1, the computational results of Ref. [13] for $E(a)$ normalized for the Casimir energy per unit area of two parallel ideal-metal planes,

$$
E_{\mathrm{im}}(a)=-\frac{\pi^{2}}{720} \frac{\hbar c}{a^{3}}
$$

are shown as black dots over the separation region from $10 \mathrm{~nm}$ to $5 \mu \mathrm{m}$. In making computations, it was assumed [13] that $\chi^{\perp}\left(i \xi, k_{\perp}\right)=\chi^{\|}\left(i \xi, k_{\perp}\right)$. The gray line shows our computational results for $E(a) / E_{\mathrm{im}}(a)$ using the polarization tensor at $T=0 \mathrm{~K}$ given by Eqs. (17) and (21). In this case, the contribution of the TE mode was calculated precisely.

As can be seen in Fig. 1, both sets of computational results are in very good agreement. This is explained by the fact that $E_{\mathrm{TM}}(a)$ contributes $99.6 \%$ of $E(a)$ and $E_{\mathrm{TE}}(a)=0.004 E(a)$ at all separation distances. Furthermore, the relative differences between the computational results of Ref. [13] for $E_{\mathrm{TE}}(a)$ (obtained under the assumption that $\chi^{\perp}=\chi^{\|}$) and our results here computed with the exact reflection coefficient $r_{\mathrm{TE}}^{(g)}$ are about $0.1 \%$. Thus, the role of the TE contribution to the Casimir energy of two graphene sheets is really negligibly small [7], and what form of the transverse density-density correlation function is used in computations is not critical. Physically, this is connected with the fact that the TE contribution is missing in the nonrelativistic limit, whereas the relativistic effects contain additional small factors of the order of $v_{F} / c$. 


\section{CASIMIR INTERACTION WITH GRAPHENE AT NONZERO TEMPERATURE}

In this section, we compare the computational results for the Casimir free energy of two graphene sheets and a freestanding graphene sheet interacting with an Au plate obtained using the approach of Ref. [13] and using the polarization tensor. All computations here are done at room temperature, $T=300 \mathrm{~K}$. In this way, we find the role of explicit dependence of the density-density correlation function and polarization tensor on the temperature.

\section{A. Two graphene sheets}

The free energy of the Casimir interaction between two sheets of undoped graphene was computed at $T=300 \mathrm{~K}$ using Eq. (1) with $r_{\mathrm{TM}, \mathrm{TE}}^{(1)}=r_{\mathrm{TM}, \mathrm{TE}}^{(2)}=r_{\mathrm{TM}, \mathrm{TE}}^{(g)}$. All computations were performed using the following two approaches: the approach of Ref. [13] using the reflection coefficients (4) and (10), expressed via the zero-temperature longitudinal density-density correlation function (19), and the approach of Ref. [12] using the reflection coefficients (5) and (11), expressed via the components of the polarization tensor (6) and (12). Within the approach of Ref. [13], the dependence of the free energy on $T$ is determined by the $T$-dependent Matsubara frequencies, whereas in the approach of Ref. [12], there is also explicit dependence of the polarization tensor on $T$ as a parameter.

In Fig. 2, we present the computational results for the Casimir free energy of two graphene sheets at $T=300 \mathrm{~K}$ as functions of separation over the interval from $10 \mathrm{~nm}$ to $1 \mu \mathrm{m}$. The results obtained using the polarization tensor at $T=300 \mathrm{~K}$ are shown as the upper solid line, and the results obtained using the longitudinal density-density correlation function (19) defined at $T=0 \mathrm{~K}$ are shown as dots. From Fig. 2, it is seen that the upper solid line deviates from dots significantly even at short separations. This is explained by the dependence of the polarization tensor on $T$ as a parameter in addition to the implicit $T$ dependence through the Matsubara frequencies. The lower (gray) solid line in Fig. 2 shows the computational

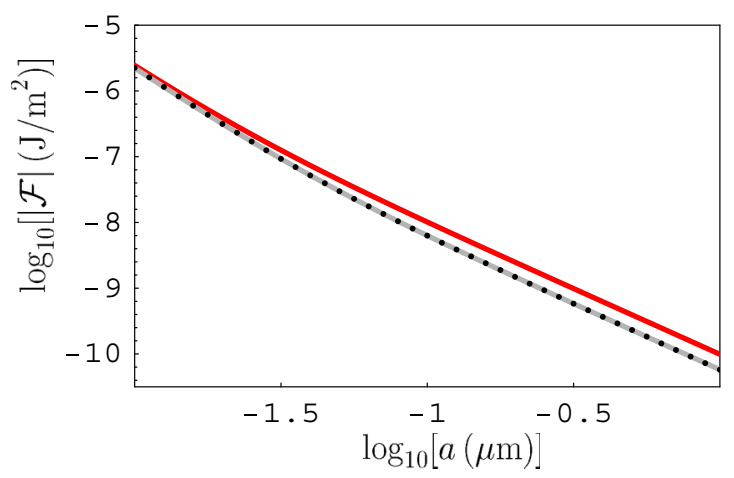

FIG. 2. (Color online) The magnitude of the Casimir free energy per unit area for two graphene sheets at $T=300 \mathrm{~K}$ is shown as a function of separation in the logarithmic scale. The upper and lower solid lines are computed using the polarization tensor at $T=300$ and $T=0 \mathrm{~K}$, respectively, whereas dots indicate the computational results using the longitudinal density-density correlation function at $T=0 \mathrm{~K}$. results obtained by means of the polarization tensor (17) and (21) at $T=0 \mathrm{~K}$. This solid line is in very good agreement with dots computed using the formalism of Ref. [13], as it should be according to the results of Secs. II and III.

Note that the dominant contribution to the free energy of the graphene-graphene interaction plotted in Fig. 2 is given by the TM mode. Thus, at $a=10 \mathrm{~nm}, \mathcal{F}_{\mathrm{TM}}=0.9965 \mathcal{F}$ and $\mathcal{F}_{\mathrm{TE}}=0.0035 \mathcal{F}$. Computations show that the contribution of the TM mode to the total free energy increases with the increase of separation. As a result, at $a=100 \mathrm{~nm}$, it holds that $\mathcal{F}_{\mathrm{TM}}=$ $0.9992 \mathcal{F}$ and, at $a=1 \mu \mathrm{m}, \mathcal{F}_{\mathrm{TM}}=0.9999 \mathcal{F}$.

It should be stressed also that the deviation between the upper and lower lines in Fig. 2 is explained entirely by the thermal dependence of the polarization tensor at zero Matsubara frequency. As was shown in Refs. [10-12] (see also Ref. [23]), contributions of all Matsubara terms with $l \geqslant 1$ are nearly the same, irrespective of whether the polarization tensor at $T=0 \mathrm{~K}$ or at $T \neq 0 \mathrm{~K}$ is used in computations.

In this respect, we remind the reader that the contribution of the zero-frequency term, $\mathcal{F}_{l=0}$, to the total free energy of two graphene sheets $\mathcal{F}$ is increasing with the increase of separation, for example, $\mathcal{F}_{l=0}=0.32 \mathcal{F}$ at $a=10 \mathrm{~nm}, \mathcal{F}_{l=0}=0.946 \mathcal{F}$ at $a=100 \mathrm{~nm}$, and $\mathcal{F}_{l=0}=0.9994 \mathcal{F}$ at $a=1 \mu \mathrm{m}$. The classical limit is already achieved at $a=400 \mathrm{~nm}$, where $\mathcal{F}_{l=0}=0.996 \mathcal{F}$. At $a \geqslant 400 \mathrm{~nm}$, the Casimir free energy shown by the upper line in Fig. 2 is given with high accuracy by the asymptotic expression [10,12,24]

$$
\mathcal{F}(a, T) \approx-\frac{k_{B} T \zeta(3)}{16 \pi a^{2}}\left[1-\frac{1}{4 \alpha \ln 2}\left(\frac{v_{F}}{c}\right)^{2} \frac{\hbar c}{a k_{B} T}\right],
$$

where $\zeta(z)$ is the Riemann zeta function.

This should be compared with the asymptotic free energy

$$
\mathcal{F}(a, T) \approx-\frac{k_{B} T}{16 \pi a^{2}} \operatorname{Li}_{3}\left(r_{0}^{(g)^{2}}\right),
$$

where $\mathrm{Li}_{3}(z)$ is the polylogarithm function. Equation (27) is obtained using the approach of Ref. [13], where the TM reflection coefficient at $\xi_{0}=0$ is defined by Eqs. (4) and (18),

$$
r_{\mathrm{TM}}^{(g)}\left(0, k_{\perp}\right) \equiv r_{0}^{(g)}=\frac{\pi e^{2}}{2 \hbar v_{F}+\pi e^{2}} .
$$

The asymptotic expression (27) is in very good agreement with the lower solid line (and dots) in Fig. 2 at $a \geqslant 400 \mathrm{~nm}$. We also notice that the contribution of the TE mode at $l=0$ to the free energy, $\mathcal{F}_{\mathrm{TE}, l=0}$, is negligibly small, as compared with the contribution of the TM mode at $l=0$ and with the total free energy, $\left|\mathcal{F}_{\mathrm{TE}, l=0}\right|<2 \times 10^{-9}\left|\mathcal{F}_{\mathrm{TM}, l=0}\right|$ and $\left|\mathcal{F}_{\mathrm{TE}, l=0}\right|<$ $1.2 \times 10^{-9}|\mathcal{F}|$, over the entire region of separations.

Now we present a more informative comparison between the approaches using the polarization tensor at $T=300 \mathrm{~K}$ and the longitudinal density-density correlation function at $T=0 \mathrm{~K}$, avoiding the use of the logarithmic scale. For this purpose, we plot in Figs. 3(a) and 3(b) the ratios of the obtained results for the free energy to the asymptotic free energy of two ideal-metal planes at high temperature, defined as [16]

$$
\mathcal{F}_{\text {im }}(a, T)=-\frac{k_{B} T \zeta(3)}{8 \pi a^{2}} .
$$

The upper and lower solid lines are computed by Eq. (1) using the polarization tensor at $T=300$ and $T=0 \mathrm{~K}$, respectively. 

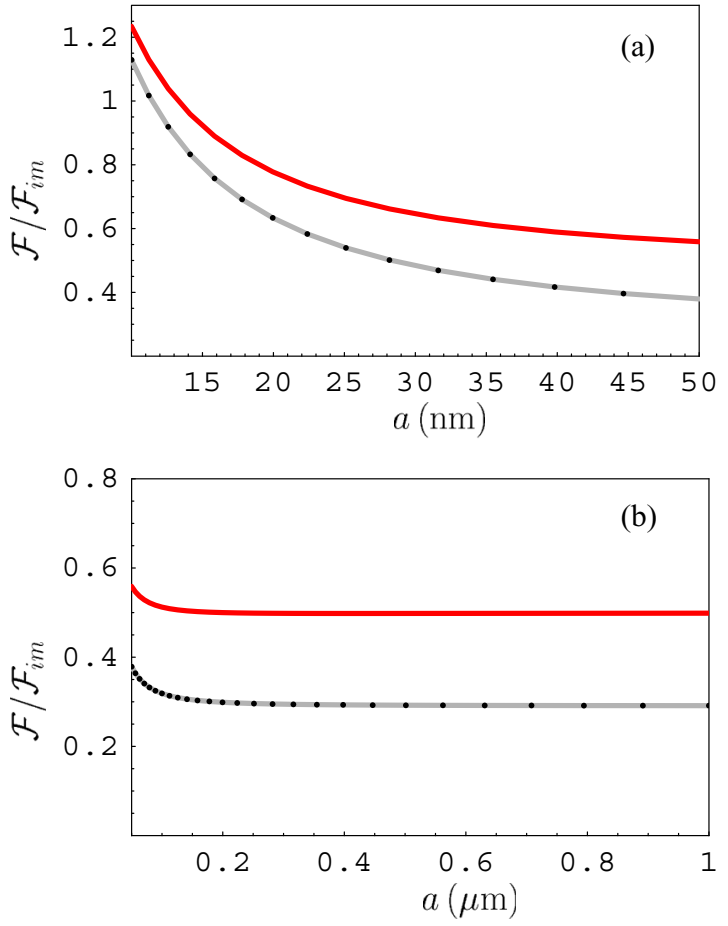

FIG. 3. (Color online) The Casimir free energy per unit area of two graphene sheets at $T=300 \mathrm{~K}$ normalized to that of two ideal-metal planes in the limit of high $T$ is computed using the longitudinal density-density correlation function at $T=0 \mathrm{~K}$ (dots), by the polarization tensor at $T=300 \mathrm{~K}$ (the upper solid line), and by the polarization tensor at $T=0 \mathrm{~K}$ (the lower solid line) over the separation regions (a) from 10 to $50 \mathrm{~nm}$ and (b) from $50 \mathrm{~nm}$ to $1 \mu \mathrm{m}$.

The dots indicate the computational results of Ref. [13] obtained at $T=300 \mathrm{~K}$ using the longitudinal density-density correlation function defined at $T=0 \mathrm{~K}$. From Fig. 3(a), it becomes clear that even at the shortest separations from 10 to $50 \mathrm{~nm}$, where in the logarithmic scale of Fig. 2 the computational results using the two approaches might seem to be very close, there are in fact large deviations, illustrating the role of explicit thermal dependence of the polarization tensor. In Fig. 3(b), plotted for the separation region from $50 \mathrm{~nm}$ to $1 \mu \mathrm{m}$, it is seen that the high-temperature limits predicted by the two approaches also differ significantly. Note that the computational results shown by the upper solid lines agree with those of Ref. [7], where the temperature dependence of the longitudinal density-density correlation function was found by scaling.

Finally, in Fig. 4, we plot by the lower solid line the relative deviation between the free energies of two graphene sheets computed using the longitudinal density-density correlation function at $T=0 \mathrm{~K}\left(\mathcal{F}_{\mathrm{dd}}\right)$ and the polarization tensor at $T=$ $300 \mathrm{~K}\left(\mathcal{F}_{\mathrm{pt}}\right)$,

$$
\delta \mathcal{F}(a, T)=\frac{\mathcal{F}_{\mathrm{dd}}(a, T)-\mathcal{F}_{\mathrm{pt}}(a, T)}{\mathcal{F}_{\mathrm{pt}}(a, T)} .
$$

As is seen in Fig. 4, at the shortest separation $a=10 \mathrm{~nm}$, the magnitude of the relative deviation $|\delta \mathcal{F}|=8.5 \%$, then it achieves the value of $|\delta \mathcal{F}|=41.2 \%$ at $a=400 \mathrm{~nm}$, and does not exceed $41.8 \%$ at all larger separations.

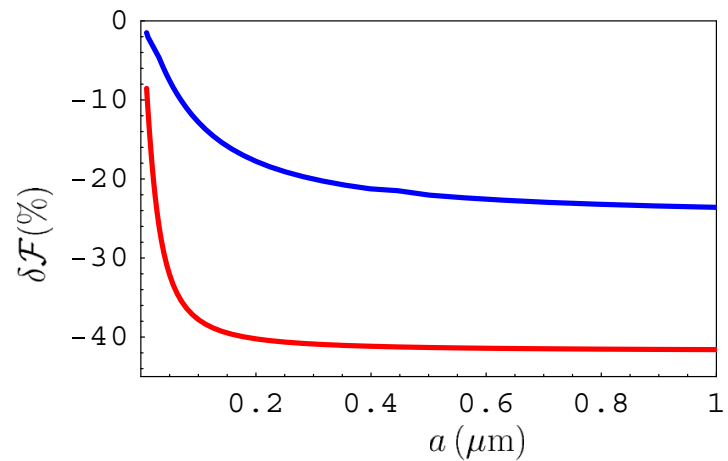

FIG. 4. (Color online) The solid lines show the relative deviations between the Casimir free energies of graphene-graphene (the lower line) and graphene-Au plate (the upper line) interactions computed using the longitudinal density-density correlation function at $T=0 \mathrm{~K}$ and the polarization tensor at $T=300 \mathrm{~K}$.

To conclude the consideration of two graphene sheets, we stress that the calculation approach using the temperaturedependent density-density correlation functions (9) and (14), found in Sec. II, and the reflection coefficients (4) and (10) lead to precisely the same results as the temperature-dependent polarization tensor.

\section{B. Graphene sheet and a gold plate}

We have calculated the Casimir free energy at $T=300 \mathrm{~K}$ for a graphene sheet interacting with an $\mathrm{Au}$ plate using the two theoretical approaches discussed above. For this purpose, Eq. (1) was used where the reflection coefficients $r_{\mathrm{TM}, \mathrm{TE}}^{(1)}=$ $r_{\mathrm{TM}, \mathrm{TE}}^{(g)}$ are defined in Secs. II and III, and $r_{\mathrm{TM}, \mathrm{TE}}^{(2)}=r_{\mathrm{TM}, \mathrm{TE}}^{(\mathrm{Au})}$ are defined as

$$
\begin{aligned}
& r_{\mathrm{TM}}^{(\mathrm{Au})}\left(i \xi_{l}, k_{\perp}\right)=\frac{\varepsilon\left(i \xi_{l}\right) q_{l}-k_{l}}{\varepsilon\left(i \xi_{l}\right) q_{l}+k_{l}}, \\
& r_{\mathrm{TE}}^{(\mathrm{Au})}\left(i \xi_{l}, k_{\perp}\right)=\frac{q_{l}-k_{l}}{q_{l}+k_{l}} .
\end{aligned}
$$

Here, $\varepsilon(\omega)$ is the frequency-dependent dielectric permittivity of $\mathrm{Au}$ and

$$
k_{l} \equiv k_{l}\left(i \xi_{l}, k_{\perp}\right)=\left[k_{\perp}^{2}+\varepsilon\left(i \xi_{l}\right) \frac{\xi_{l}^{2}}{c^{2}}\right]^{1 / 2} .
$$

The dielectric permittivity of Au at the imaginary Matsubara frequencies was obtained from the experimental optical data [25] for the imaginary part of the dielectric function by means of the Kramers-Kronig relation. The data were previously extrapolated to lower frequencies by means of the Drude model. In this paper, the data for $\varepsilon\left(i \xi_{l}\right)$ from Ref. [13] have been used in the computations. The alternative extrapolation of the optical data by means of the plasma model leads to a maximum relative deviation in the obtained free energy equal to $0.8 \%$ at the shortest separation, $a=10 \mathrm{~nm}$, and to smaller deviations at larger separations. As noted in Ref. [11], for a graphene-metal interaction, the Casimir free energy and pressure do not depend on what model of metal (Drude or plasma) is used to describe the metal. For two metallic plates, there are large differences in the results 


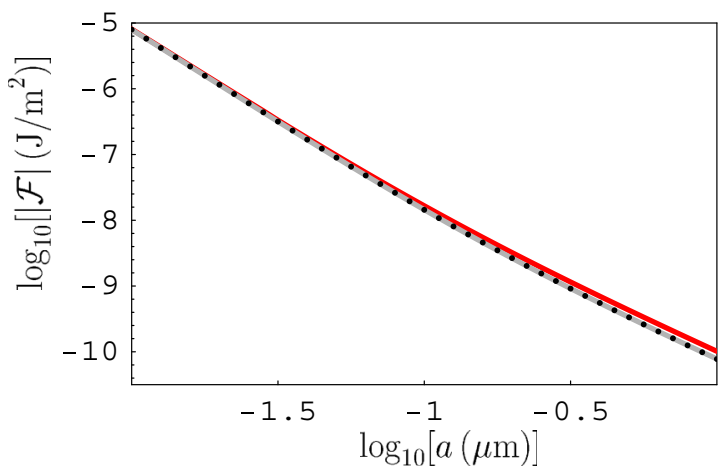

FIG. 5. (Color online) The magnitude of the Casimir free energy per unit area for a graphene sheet and an Au plate at $T=300 \mathrm{~K}$ is shown as a function of separation in the logarithmic scale. The upper and lower solid lines are computed using the polarization tensor at $T=300$ and $T=0 \mathrm{~K}$, respectively, whereas dots indicate the computational results using the longitudinal density-density correlation function at $T=0 \mathrm{~K}$.

obtained using the Drude or plasma models [26] due to the contribution of the TE mode, which is negligibly small for a graphene sheet.

In Fig. 5, the computational results for the Casimir free energy of a graphene sheet interacting with an $\mathrm{Au}$ plate at $T=300 \mathrm{~K}$ are presented as functions of separation in the region from $10 \mathrm{~nm}$ to $1 \mu \mathrm{m}$. The upper and lower solid lines indicate the results obtained using Eq. (1) and the polarization tensor at $T=300$ and $T=0 \mathrm{~K}$, respectively. The dots show the results [13] computed from the longitudinal density-density correlation function (19) at $T=0 \mathrm{~K}$. As is seen in Fig. 5, dots are in agreement with the lower solid line, but deviate significantly from the upper one. This demonstrates the important role of the explicit dependence of the polarization tensor on the temperature.

As in the case of two graphene sheets, the dominant contribution to $\mathcal{F}$ is given by the TM mode. Here, however, the ratio $\mathcal{F}_{\mathrm{TM}} / \mathcal{F}$ is not a monotonous function of $a$. Thus, at $a=10 \mathrm{~nm}, \mathcal{F}_{\mathrm{TM}}=0.983 \mathcal{F}$ and, at $a=100 \mathrm{~nm}$, the TM contribution achieves its minimum value $\mathcal{F}_{\mathrm{TM}}=0.961 \mathcal{F}$. With further increase of separation, $\mathcal{F}_{\mathrm{TM}}$ increases to $0.983 \mathcal{F}$, $0.992 \mathcal{F}$, and $0.998 \mathcal{F}$ at $a=500 \mathrm{~nm}, 1 \mu \mathrm{m}$, and $2 \mu \mathrm{m}$, respectively. Similar to the case of two graphene sheets, the difference between the upper and lower solid lines is explained by the explicit thermal dependence of the polarization tensor at zero Matsubara frequency [10,11].

For a graphene sheet interacting with an $\mathrm{Au}$ plate, the contribution of the zero Matsubara frequency to the total free energy increases with separation slower than for two graphene sheets. Thus, at $a=10$ and $100 \mathrm{~nm}$, one obtains $\mathcal{F}_{l=0}=0.11 \mathcal{F}$ and $0.58 \mathcal{F}$, respectively. At $a=1 \mu \mathrm{m}, \mathcal{F}_{l=0}=0.97 \mathcal{F}$, and, at $a=1.6 \mu \mathrm{m}$, the classical limit is achieved: $\mathcal{F}_{l=0}=0.99 \mathcal{F}$. At this and larger separations, the Casimir free energy per unit area is given by $[10,12]$

$$
\mathcal{F}(a, T) \approx-\frac{k_{B} T \zeta(3)}{16 \pi a^{2}}\left[1-\frac{1}{8 \alpha \ln 2}\left(\frac{v_{F}}{c}\right)^{2} \frac{\hbar c}{a k_{B} T}\right] .
$$

The calculation approach using the longitudinal densitydensity correlation function defined at zero temperature
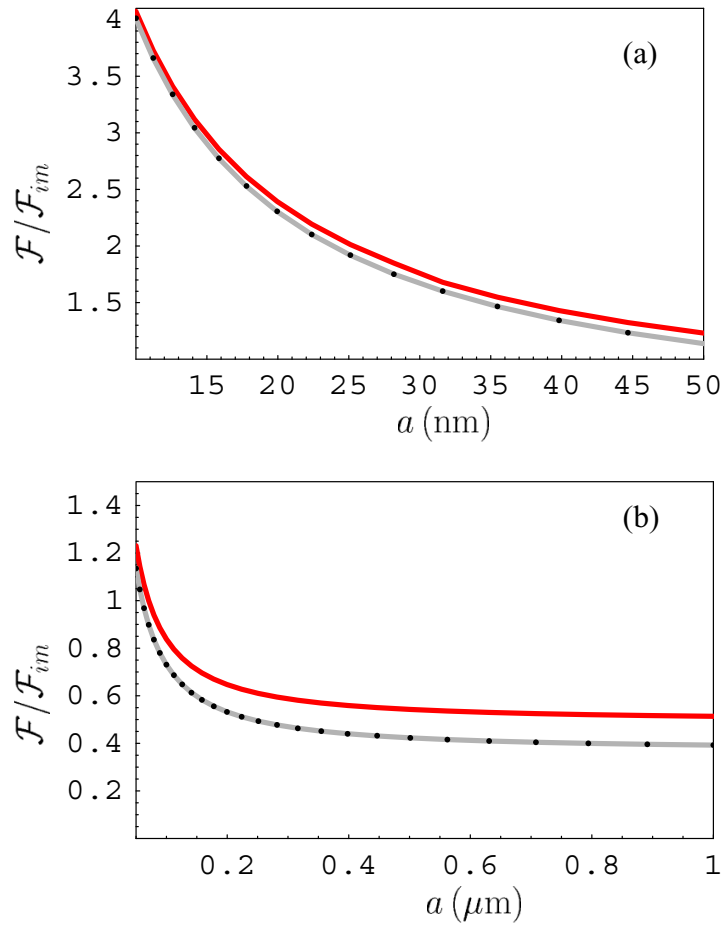

FIG. 6. (Color online) The Casimir free energy per unit area of a graphene sheet and an Au plate at $T=300 \mathrm{~K}$ normalized to that of two ideal-metal planes in the limit of high $T$ is computed using the longitudinal density-density correlation function at $T=0 \mathrm{~K}$ (dots), by the polarization tensor at $T=300 \mathrm{~K}$ (the upper solid line), and by the polarization tensor at $T=0 \mathrm{~K}$ (the lower solid line) over the separation regions (a) from 10 to $50 \mathrm{~nm}$ and (b) from $50 \mathrm{~nm}$ to $1 \mu \mathrm{m}$.

describes the reflection coefficient on graphene at $\xi_{0}=0$ by Eq. (28). Taking into account that for $\mathrm{Au}, r_{\mathrm{TM}}^{(\mathrm{Au})}\left(0, k_{\perp}\right)=1$, the classical limit is obtained in the form

$$
\mathcal{F}(a, T) \approx-\frac{k_{B} T}{16 \pi a^{2}} \mathrm{Li}_{3}\left(r_{0}^{(g)}\right) .
$$

This asymptotic expression is in very good agreement with the computational results of Ref. [13] at $a \geqslant 1.6 \mu \mathrm{m}$.

To avoid the use of the logarithmic scale, in Figs. 6(a) and $6(\mathrm{~b})$, we plot the ratios of the computed free energies of the graphene-Au plate interaction to the asymptotic free energy of two ideal-metal planes (29). The upper and lower solid lines are computed by Eq. (1) using the polarization tensor defined at $T=300$ and $T=0 \mathrm{~K}$, respectively. The dots are computed in Ref. [13] at $T=300 \mathrm{~K}$ using the longitudinal density-density correlation function defined at $T=0 \mathrm{~K}$. From Figs. 6(a) and 6(b), it is seen that there are significant deviations between the upper line, on the one hand, and the lower line and dots, on the other hand, at both short and relatively large separations. At $a \geqslant 1.6 \mu \mathrm{m}$, the discrepancy between the theoretical predictions of the two approaches is illustrated by Eqs. (33) and (34).

The relative deviation (30) between the Casimir free energies of the graphene-Au plate interaction computed using the two approaches is shown by the upper line in Fig. 4. Here, the magnitude of the relative deviation is equal to $|\delta \mathcal{F}|=1.5 \%$ at $a=10 \mathrm{~nm}$, achieves $|\delta \mathcal{F}|=24 \%$ at $a=1.5 \mu \mathrm{m}$, and does not exceed $24.5 \%$ at larger separations. This means that large 
thermal effects inherent to graphene are less pronounced in the graphene-Au plate configuration, as compared to the case of two graphene sheets.

Similar to two graphene sheets, for a graphene interaction with an Au plate, the theoretical predictions using the polarization tensor at $T \neq 0$ are in full agreement with the respective predictions using the $T$-dependent density-density correlation function defined in Eqs. (9) and (14).

\section{CONCLUSIONS AND DISCUSSION}

In the foregoing, we have performed a comparison study of two approaches used to calculate the van der Waals and Casimir interaction between two graphene sheets and between a graphene sheet and a metal plate. One of these approaches is based on the use of the polarization tensor. All of its components are found [10] at any temperature. The other approach is based on the use of the density-density correlation function. Only the longitudinal version of this function was available in the literature and only at zero temperature. Because of this, previous calculations estimated the TE contribution to the free energy as negligibly small and either modeled the temperature dependence of the correlation function by means of scaling between two asymptotic regimes [7] or argued that this dependence is not essential [13].

We have shown that at zero temperature the approaches using the polarization tensor and the standard longitudinal density-density correlation function lead to almost coinciding computational results. The coincidence becomes exact if, in the calculation of negligibly small contribution of the TE mode, one replaces the longitudinal density-density correlation function at $T=0 \mathrm{~K}$ with the transverse one. We have provided an explicit expression for this function.

Computations at nonzero temperature using the polarization tensor with an explicit thermal dependence demonstrate significant deviations from the computational results using the density-density correlation function at $T=0 \mathrm{~K}$. The latter include only an implicit dependence of the Casimir free energy on the temperature through the Matsubara frequencies. It was shown that for graphene-graphene and graphene-Au plate interactions, the free energies obtained using this approach deviate from those calculated using the temperature-dependent polarization tensor up to $41.8 \%$ and $24.5 \%$, respectively. However, the computational results obtained using the zerotemperature density-density correlation function are reproduced when the polarization tensor defined at $T=0 \mathrm{~K}$ is used. Similar to the case of zero temperature, at $T \neq 0$ $\mathrm{K}$, the contribution of the TE mode of the electromagnetic field to the Casimir free energy is shown to be negligibly small for both graphene-graphene and graphene-Au plate systems.

We have performed a comparison between the exact TM and TE reflection coefficients expressed via the components of the polarization tensor, on the one hand, and via the longitudinal and transverse density-density correlation functions, on the other hand. In this way, we have found explicit expressions for both longitudinal and transverse density-density correlation functions at any nonzero temperature. In the limiting case of vanishing temperature, our temperature-dependent longitudinal density-density correlation function goes into the well-known classical result. The computational results for graphene-graphene and graphene-Au plate Casimir interactions obtained using the temperature-dependent densitydensity correlation functions found by us exactly coincide with those obtained using the temperature-dependent polarization tensor.

One can conclude that an equivalence of the two approaches to the calculation of the van der Waals and Casimir forces in layered systems including graphene, demonstrated in this paper, provides a reliable foundation for the comparison between experiment and theory.
[1] A. H. Castro Neto, F. Guinea, N. M. R. Peres, K. S. Novoselov, and A. K. Geim, Rev. Mod. Phys. 81, 109 (2009).

[2] M. S. Dresselhaus, Phys. Stat. Solidi (B) 248, 1566 (2011).

[3] A. A. Banishev, H. Wen, J. Xu, R. K. Kawakami, G. L. Klimchitskaya, V. M. Mostepanenko, and U. Mohideen, Phys. Rev. B 87, 205433 (2013).

[4] J. F. Dobson, A. White, and A. Rubio, Phys. Rev. Lett. 96, 073201 (2006).

[5] Bo E. Sernelius, Europhys. Lett. 95, 57003 (2011).

[6] M. Bordag, I. V. Fialkovsky, D. M. Gitman, and D. V. Vassilevich, Phys. Rev. B 80, 245406 (2009).

[7] G. Gómez-Santos, Phys. Rev. B 80, 245424 (2009).

[8] D. Drosdoff and L. M. Woods, Phys. Rev. B 82, 155459 (2010).

[9] V. Svetovoy, Z. Moktadir, M. Elvenspoek, and H. Mizuta, Europhys. Lett. 96, 14006 (2011).

[10] I. V. Fialkovsky, V. N. Marachevsky, and D. V. Vassilevich, Phys. Rev. B 84, 035446 (2011).

[11] M. Bordag, G. L. Klimchitskaya, and V. M. Mostepanenko, Phys. Rev. B 86, 165429 (2012).
[12] G. L. Klimchitskaya and V. M. Mostepanenko, Phys. Rev. B 87, 075439 (2013).

[13] Bo E. Sernelius, Phys. Rev. B 85, 195427 (2012).

[14] E. M. Lifshitz and L. P. Pitaevskii, Statistical Physics, Part II (Pergamon, Oxford, 1980).

[15] Bo E. Sernelius, Surface Modes in Physics (Wiley-VCH, Berlin, 2001).

[16] M. Bordag, G. L. Klimchitskaya, U. Mohideen, and V. M. Mostepanenko, Advances in the Casimir Effect (Oxford University Press, Oxford, 2009).

[17] L. A. Falkovsky and S. S. Pershoguba, Phys. Rev. B 76, 153410 (2007).

[18] T. Stauber, N. M. R. Peres, and A. K. Geim, Phys. Rev. B 78, 085432 (2008).

[19] Kenneth W.-K. Shung, Phys. Rev. B 34, 979 (1986).

[20] J. Gonzáles, F. Guinea, and M. A. H. Vozmediano, Nucl. Phys. 424, 595 (1994).

[21] B. Wunsch, T. Stauber, F. Sols, and F. Guinea, New J. Phys. 8, 318 (2006).

[22] N. M. R. Peres, F. Guinea, and A. H. Castro Neto, Phys. Rev. B 73, 125411 (2006). 
[23] M. Chaichian, G. L. Klimchitskaya, V. M. Mostepanenko, and A. Tureanu, Phys. Rev. A 86, 012515 (2012).

[24] G. L. Klimchitskaya and V. M. Mostepanenko, Phys. Rev. B 89, 035407 (2014).
[25] Handbook of Optical Constants of Solids, edited by E. D. Palik (Academic, New York, 1985).

[26] G. L. Klimchitskaya, U. Mohideen, and V. M. Mostepanenko, Rev. Mod. Phys. 81, 1827 (2009). 